\title{
SINTONIA ÓTIMA PID COM ALGORITMO GENÉTICO PARA MINIMIZAR VARIAÇÕES DA AÇÃO DE CONTROLE*
}

\author{
Diego Santana Torga ${ }^{1}$ \\ Cristiano Lopes da Silva ${ }^{1}$ \\ Thiago Antonio Melo Euzébio ${ }^{2}$ \\ Frederico Gadelha Guimarães ${ }^{3}$
}

\section{Resumo}

É comum encontrar sintonia de controladores PID que não levam em consideração o sinal de controle aplicado a variável manipulada. Portanto, variações rápidas e amplitudes elevadas podem ser geradas, que, na prática, pode ser inviáveis, devido à limitação física de atuadores e equipamentos industriais. Nesse artigo é desenvolvido um método de sintonia em que os parâmetros do PID são calculados por meio do Algoritmo Genético. A função de custo proposta penaliza tanto a amplitude do erro de controle quanto à variação da variável manipulada. A estabilidade é garantida com as restrições de robustez sobre o pico das funções sensibilidades. Exemplos simulados demonstram as vantagens da sintonia proposta. Palavras-chave: Controle de Processos; Algoritmo Genético; Otimização; PID.

\section{OPTIMUM PID TUNING WITH GENETIC ALGORITHM TO MINIMIZE CONTROL ACTION VARIATIONS}

\section{Abstract}

It is common to find PID controllers tuning that do not consider the control signal applied to the manipulated variable. Therefore, rapid variations and high amplitudes can be generated, which, in practice, may be unfeasible due to physical limitation of the actuators and industrial equipment. In this article it is developed a tuning method which the PID parameters are calculated by means the Genetic Algorithm. The proposed cost function penalizes both the control error amplitude and the manipulated variable variation. The stability is guaranteed with robustness constraints over the peak sensitivities functions. Simulated examples demonstrate the advantages of the proposed tuning.

Keywords: Process Control; Genetic Algorithm; Optimization; PID

1 Graduação em Engenharia de Controle e Automação (UFOP) e discente do Mestrado Profissional em Instrumentação, Controle e Automação de Processos de Mineração, Instituto Tecnológico Vale (ITV) / UFOP, Ouro Preto, MG, Brasil.

2 Graduação, mestrado e doutorado em Engenharia Elétrica (UFCG). Atualmente é pesquisador no Instituto Tecnológico Vale (ITV), Ouro Preto, MG, Brasil.

3 Graduação, mestrado e doutorado em Engenharia Elétrica (UFMG). Atualmente é professor adjunto do Departamento de Engenharia Elétrica da UFMG, Belo Horizonte, MG, Brasil. 


\section{INTRODUÇÃO}

Os controladores proporcional-integral-derivativo (PID) são os mais aceitos na indústria de processos. Mais de $90 \%$ das malhas de controle utilizam esses controladores [1]. As principais razões para o PID ser amplamente utilizado são: estrutura simples, robustez, fácil entendimento em relação à maioria dos controladores avançados, simplicidade de implementação prática e poucos parâmetros a serem sintonizados. Além disso, possui a característica de eliminar o erro em regime permanente por meio da ação integral e poder "antecipar" a ação de controle por meio da ação derivativa, afim de que o processo reaja mais rápido. Apesar dessas vantagens, ainda existe na indústria grande quantidade de malhas de controle operando abaixo do desempenho esperado e, um dos motivos dominantes é a sintonia ruim dos controladores [2].

Para sintonizar controladores PID, vários métodos clássicos têm sido formulados. Alguns desses métodos podem ser encontrados em [3], [4], [5] e [6]. Em comum, esses métodos têm as vantagens de serem simples, exigirem poucas informações do processo e atenderem a um conjunto de modelos de processos comuns na indústria. No entanto, podem produzir sintonias agressivas e margens de estabilidade pequena, o que na prática não é interessante, devido às não linearidades do processo. Além disso, a maioria dos processos industriais não necessita de uma resposta muito rápida e oscilatória [7].

Com o avanço tecnológico, principalmente na área da eletrônica e da informática, métodos de otimização para projeto de controladores PID têm sido formulados. Esses métodos permitem que especificações de desempenhos, níveis de robustez, limites operacionais de atuadores, dentre outros, sejam garantidos, o que nos métodos clássicos pode não ser atendidos. Para solução por meio desses métodos existem diversas ferramentas, entre as quais podemos citar o Algoritmo Genético (AG). O AG vem sendo muito utilizado, o que é justificável pela sua relativa simplicidade de implementação, flexibilidade e eficácia em realizar busca global em ambientes adversos [8]. Em [9] é apresentada a aplicação do AG na sintonia de quatro controladores $\mathrm{PI}$, para um processo não linear, sob as especificações de desempenho como o IAE e o tempo de resposta e, especificações de robustez como erro do modelo e ruído de entrada. Já em [10] é utilizado o AG MRCD (projeto de controle robusto multiobjectivo). Neste trabalho a função de custo compreende a rejeição à perturbação de carga, a robustez às incertezas do modelo e o rastreamento da referência, além da única restrição aplicada sob o limite do atuador. Em particular, diversos métodos de sintonia PID baseados em otimização não consideram as variações da ação de controle na função de custo. É comum encontrar resultados de sintonia que obtêm boas respostas simuladas, mas inviáveis em aplicações práticas, devido à limitação física de atuadores e equipamentos industriais.

Neste artigo, o AG é utilizado como uma ferramenta no problema de otimização para sintonia de controladores PID. O objetivo é considerar questões como variações da ação de controle e máximo sobressinal durante sintonia. Além disso, restrições de robustez são aplicadas sobre as funções de sensibilidades. Exemplos simulados demonstram as vantagens da sintonia proposta.

O trabalho está organizado da seguinte forma. Seção 2 apresenta a formulação do problema. Na Seção 3 são apresentadas à especificação de desempenho, as restrições de robustez e sobressinal e a formulação do problema de otimização. Fundamentos do Algoritmo Genético são apresentados na Seção 4. Exemplos 
simulados são conduzidos na Seção 5. Análise dos resultados é apresentada na Seção 6 e na Seção 7 a conclusão do trabalho.

\section{FORMULAÇÃO DO PROBLEMA}

Considere o sistema de controle (SISO) em malha fechada da Figura 1, no qual G(s) representa o modelo do processo e $\mathrm{C}(\mathrm{s})$ o controlador. O único sinal de entrada é o sinal de referência $R(s)$. Os sinais de saída são a ação de controle $U(s)$, a variável de processo $\mathrm{Y}(\mathrm{s})$ e o erro $\mathrm{E}(\mathrm{s})=\mathrm{R}(\mathrm{s})-\mathrm{Y}(\mathrm{s})$.

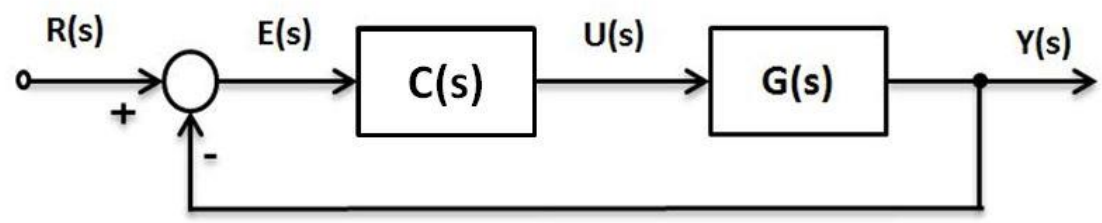

Figura 1. Diagrama de bloco de um sistema de controle SISO em malha fechada

A função de transferência do controlador $C(s)$ na formulação PID é definida conforme Equação (1).

$$
C(s)=K_{p}+\frac{K_{i}}{s}+K_{d} s
$$

Onde: $K_{p}$ é o ganho proporcional, $K_{i}$ o ganho integral e $K_{d}$ o ganho derivativo.

\section{MÉTODO DE SINTONIA BASEADO EM PROBLEMA DE OTIMIZAÇÃO}

Nesta seção é apresentado o método proposto de sintonia PID. Esse método é estruturado a partir de uma função de custo de desempenho que o problema busca minimizar e três restrições.

\subsection{Função de Custo}

A função de custo de desempenho é composta pelo somatório do erro e pelo somatório da diferença entre a ação de controle e ação de controle em estado estacionário, devido a uma variação em degrau unitário na referência. Conforme apresentado na Equação (2).

$$
J=\alpha \sum_{i=1}^{N}|e(i)|+\beta \sum_{i=2}^{N}\left|u(i-1)-u_{e e}\right|
$$

Onde: $\mathrm{N}$ é o instante que o erro é zero, $\alpha$ e $\beta$ são constantes, $u$ é o valor da ação de controle e $u_{e e}$ a ação de controle em estado estacionário.

\subsection{Conjunto de Restrições}

Para muitos processos na indústria, é comum a operação em diversas condições de funcionamento. Assim, a robustez é uma característica importante do sistema de controle em malha fechada. Há duas medidas clássicas muito utilizadas como 
medidas de robustez, margem de ganho $g_{m}$ e margem de fase $\varphi_{m}$. No entanto, as duas primeiras restrições aplicadas são: na Equação (3) a função sensibilidade máxima

$$
\|S\|_{\infty}=\max _{w}\left|\frac{1}{1+G(j w) C(j w)}\right| \leq M_{s}
$$

e na Equação (4) a função sensibilidade complementar máxima

$$
\|T\|_{\infty}=\max _{w}\left|\frac{G(j w) C(j w)}{1+G(j w) C(j w)}\right| \leq M_{t}
$$

as quais tornaram-se cada vez mais aceitas como medidas exclusivas de robustez e são utilizadas em trabalhos como [11] e [12].

Uma interpretação geométrica das restrições em (3) e (4), é que estas formam duas circunferências que englobam o ponto crítico $(-1,0)$ no diagrama de Nyquist. Assim, as exigências de que a sensibilidade máxima seja menor que $M_{s}$ e a sensibilidade complementar máxima seja menor que $M_{t}$, implicam que a função de transferência de ganho de malha $\mathrm{C}(\mathrm{s}) \mathrm{G}(\mathrm{s})$ deve estar fora das circunferências, como mostrado no exemplo da Figura 2.

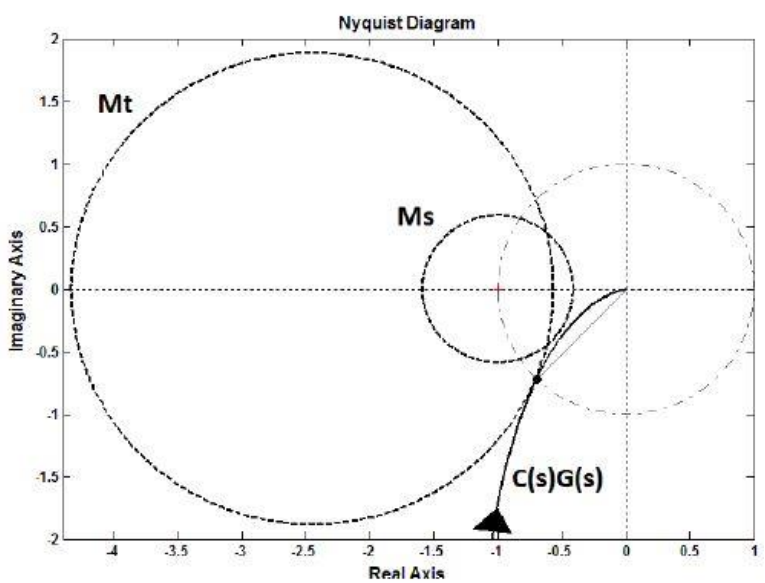

Figura 2. Diagrama de Nyquist para o ganho de malha C(s)G(s) com as restrições $\boldsymbol{M}_{\boldsymbol{s}}=1,7$ e $\boldsymbol{M}_{\boldsymbol{t}}=$ 1,3 .

A terceira restrição escolhida trata-se de um limite superior na variável de processo para uma resposta ao degrau unitário na referência. A ideia é que seja possível já na sintonia definir limite máximo de sobressinal, conforme definido na Equação (5).

$$
P V_{\max } \leq a
$$

Onde: $a$ é uma constante.

\subsection{Problema de Otimização}

Com base na função de custo definida em (2) e as restrições definidas em (3), (4) e (5), o seguinte problema de otimização é formulado em (6). 
sujeito a:

$$
\min _{K_{p}, K_{i}, K_{d}} J
$$

$$
\begin{gathered}
\|S\|_{\infty} \leq M_{s}, \\
\|T\|_{\infty} \leq M_{t}, \\
P V_{\text {max }} \leq a .
\end{gathered}
$$

\section{ALGORITMO GENÉTICO}

Algoritmo Genético ( $A G)$ são técnicas de otimização baseadas no mecanismo da evolução das espécies. Em AG uma população inicial de possíveis soluções é gerada para o problema descrito, assim, à medida que o problema evolui com os operadores genéticos, existe uma tendência, de que na média, os novos indivíduos possuam soluções melhores que a geração anterior. Dessa forma, o processo evolutivo direciona a busca para regiões de melhores soluções, de tal maneira que a melhor solução seja encontrada.

Introduzido inicialmente por Holland [13], o AG tem sido amplamente utilizado, especialmente em aplicações de engenharia. Uma de suas características principais, em contraste a outros métodos de otimização, é preservar uma população de soluções potenciais até encontrar uma solução aceitável. Em [8] são apresentadas as componentes básicas que constituem o AG e que são descritas a seguir. Além disso, na Figura 3 é apresentado o fluxograma genérico do AG.

\subsection{Representação Genética}

É a representação do problema a ser resolvido de maneira que seja compreendido por computadores. Neste caso, as variáveis do problema são formadas por um ou mais cromossomos (indivíduo) e, em geral, são implementadas como vetores, sendo cada posição do vetor chamada de gene. Estas podem ser codificadas de forma binária, real ou inteira.

\subsection{População Inicial}

São as possíveis soluções do problema e que podem ser obtidas de forma aleatória ou por meio de uma heurística. Uma boa definição da população quanto ao seu tamanho e limites do espaço de soluções são importantes para a eficiência do AG.

\subsection{Função Objetiva}

É a função que o problema busca minimizar ou maximizar.

\subsection{Métodos de Seleção}

São métodos utilizados para selecionar os indivíduos que serão submetidos aos operadores genéticos. Esses métodos, em sua maioria, avaliam o valor de aptidão (valor da função objetivo) de cada indivíduo para seleção. Dentre vários métodos de seleção podemos citar: roleta, torneio e amostragem universal estocástica (SUS).

\subsection{Operadores Genéticos}


Os operadores genéticos reproduzem o mecanismo de evolução dos indivíduos. Os mais usuais são: Cruzamento e Mutação.

\subsubsection{Cruzamento}

Nesta, dois indivíduos são combinados para formar novos dois indivíduos, de tal forma que, a hereditariedade seja transferida para as gerações atuais. Dessa forma, pode-se gerar uma população de soluções melhores a cada geração. Os tipos mais comuns de cruzamentos encontrados são: cruzamento uniforme e de um ou dois pontos de cortes.

\subsubsection{Mutação}

São variações aleatórias de partes (genes) do cromossomo de um indivíduo na população e que geram novos indivíduos. A mutação reintroduz a diversidade genética na população e é essencial para que novas populações de soluções sejam encontradas, auxiliando o algoritmo a buscar os mínimos ou máximos globais da função objetivo.

\subsection{Critérios de Parada}

São critérios que delimitam sua busca e finalizam a execução do AG. Dentre alguns critérios podemos citar: número de gerações, tempo de execução ou quando uma população não melhora em sucessivas gerações.

\subsection{Parâmetros do AG}

Embora o AG possua a característica de encontrar boas soluções de forma eficaz, o mesmo é sensível a alguns parâmetros que são definidos na sua configuração, sendo esses: tamanho da população, número de gerações e taxa de cruzamento e mutação.

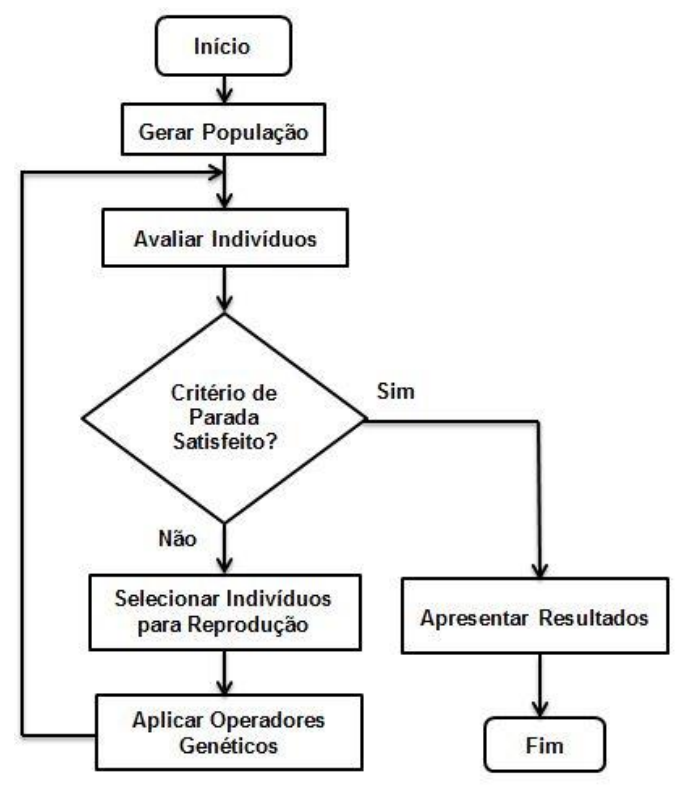

Figura 3. Fluxograma genérico do AG 


\section{RESULTADOS}

Nesta seção são apresentados dois exemplos simulados com a sintonia proposta, comparadas com a sintonia do trabalho de Åström e Hägglund [14]. A escolha por esse trabalho é devido o mesmo ser um problema baseado em otimização e utilizar restrição de robustez sobre o pico da função sensibilidade máxima, o que também é realizado neste trabalho. Para resolução do problema de otimização formulado em (6), é utilizado o software Matlab ${ }^{\circledR}$ e sua ferramenta de algoritmo genético. O AG foi configurado conforme apresentado a seguir.

$\checkmark$ População igual a 30;

$\checkmark$ Geração igual a 10;

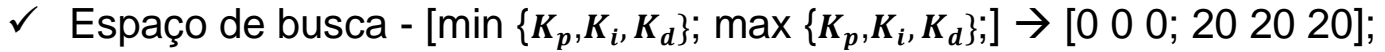

$\checkmark$ Taxa de cruzamento igual 0,7 ;

$\checkmark$ Seleção por torneio;

$\checkmark$ Cruzamento com um ponto de corte;

$\checkmark$ Critério de parada por número de geração ou quando a média de aptidão da população não melhora em sucessivas gerações.

\subsection{Exemplo 1 - Sintonia PID Aplicada ao Modelo Linear de Quarta Ordem}

Foi considerado o seguinte modelo de quarta ordem, apresentado em (7)

$$
G(s)=\frac{1}{(s+1)^{4}}
$$

utilizado no trabalho de Åström e Hägglund [14]. O problema de otimização desenvolvido por estes é conhecido como MIGO ( $M$ constrained Integral Gain Optimization), no qual a única restrição utilizada é $M_{s}=1,4$. Para o problema de otimização proposto, as constantes $\alpha$ e $\beta$, na função de custo $\mathrm{J}$ em (2), foram consideradas iguais a um, além das restrições de $M_{s}=1,4, M_{t}=1,5$ e $a=1$. Os resultados das sintonias são apresentados na Tabela 1.

Tabela 1. Parâmetros Controlador PID

\begin{tabular}{cccc}
\hline Sintonia & $\boldsymbol{K}_{\boldsymbol{p}}$ & $\boldsymbol{K}_{\boldsymbol{i}}$ & $\boldsymbol{K}_{\boldsymbol{d}}$ \\
\hline MIGO & 1,305 & 0,758 & 1,705 \\
Proposta & 0,926 & 0,256 & 1,028 \\
\hline
\end{tabular}

$\mathrm{Na}$ Figura 4 são apresentadas as respostas para as duas sintonias quando submetidas a um degrau unitário na referência. Como pode ser observado, as duas sintonias se acomodam próximas do mesmo instante de tempo. No entanto, a sintonia MIGO produz um sobressinal de aproximadamente $18 \%$ e um pico na ação de controle de cerca de $60 \%$ a mais que a sintonia Proposta. 

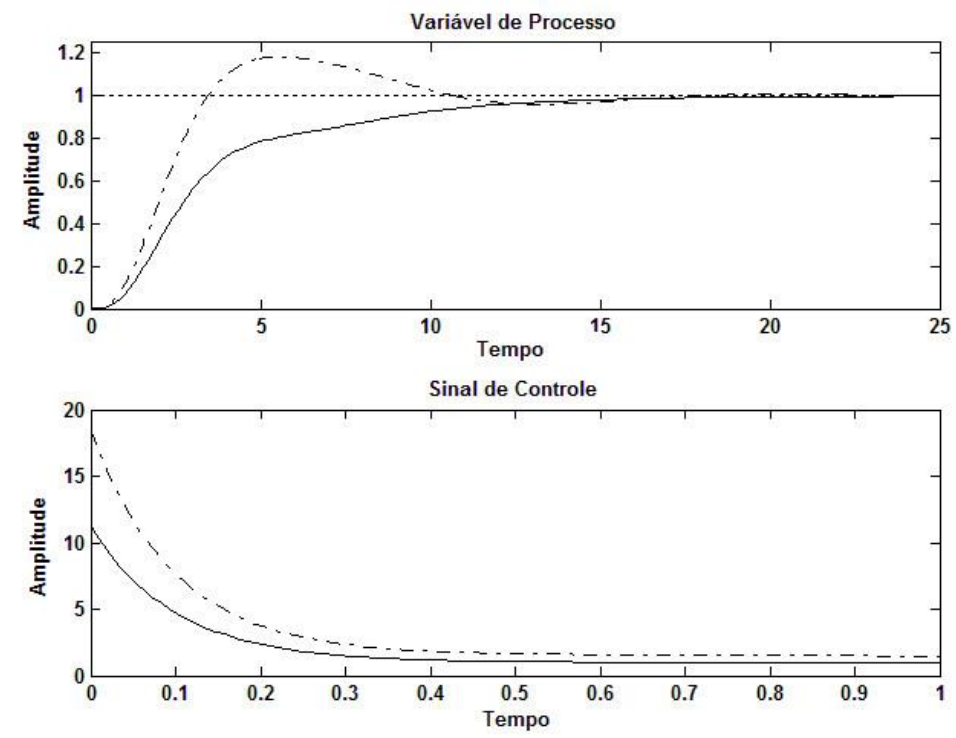

Figura 4. Resposta em malha fechada para um degrau unitário na referência. Controlador MIGO (---) e controlador Proposto (-). Escalas de tempo diferentes para variável de processo e sinal de controle.

\subsection{Exemplo 2 - Sintonia PI Aplicada a Modelos Lineares de Primeira Ordem mais Atraso}

Neste exemplo são considerados dois modelos lineares de primeira ordem mais atraso, os quais são apresentados em (8).

$$
G_{1}(s)=\frac{6 e^{-0,2 s}}{40 s+1} ; G_{2}(s)=\frac{2 e^{-0,5 s}}{80 s+1}
$$

Para comparação é utilizada a sintonia AMIGO (Approximate MIGO) para controladores Pl. O problema de otimização em (6) é configurado conforme a seguir. $\mathrm{Na}$ função de custo $\mathrm{J}$ em (2), após algumas simulações, definiu-se: $\alpha=30$ e $\beta=1$. O conjunto de restrições foi mantido conforme apresentado no Exemplo 1. Os parâmetros dos controladores PI são apresentados na Tabela 2.

Tabela 2. Parâmetros Controlador PI

\begin{tabular}{cccc}
\hline Modelo & Sintonia & $\boldsymbol{K}_{\boldsymbol{p}}$ & $\boldsymbol{K}_{\boldsymbol{i}}$ \\
\hline \multirow{2}{*}{$\boldsymbol{G}_{\mathbf{1}}(\boldsymbol{s})$} & AMIGO & 11,527 & 4,569 \\
& Proposta & 4,658 & 0,118 \\
\multirow{2}{*}{$\boldsymbol{G}_{\mathbf{2}}(\boldsymbol{s})$} & AMIGO & 27,581 & 4,434 \\
& Proposta & 5,179 & 0,018 \\
\hline
\end{tabular}

A comparação é apresentada na Figura 5 para um degrau unitário na referência de $G_{1}(s)$ e $G_{2}(s)$. Ao analisar os parâmetros (Tabela 2) dos controladores PI, tanto para $G_{1}(s)$ quanto para $G_{2}(s)$, já é esperado que a sintonia AMIGO atinja a referência em um tempo menor, já que possuem valores maiores. No entanto, a resposta em $G_{1}(s)$ para a variável de processo à sintonia Proposta, é mais suave, não possui sobressinal e seu tempo de acomodação é menor. $\mathrm{Em} G_{2}(s)$, ao realizar a mesma avaliação, a sintonia Proposta continua sem sobressinal, mas seu tempo de 
acomodação é maior. Porém, ao analisar o erro relativo com a referência esse pode ser insignificante, a depender do processo controlado. Além disso, a sintonia Proposta necessita de uma ação de controle menor, como pode ser observado. Em $G_{1}(s)$ e $G_{2}(s)$ essa variação e amplitude do sinal de controle é de aproximadamente $62 \%$ e $82 \%$ menor, respectivamente.
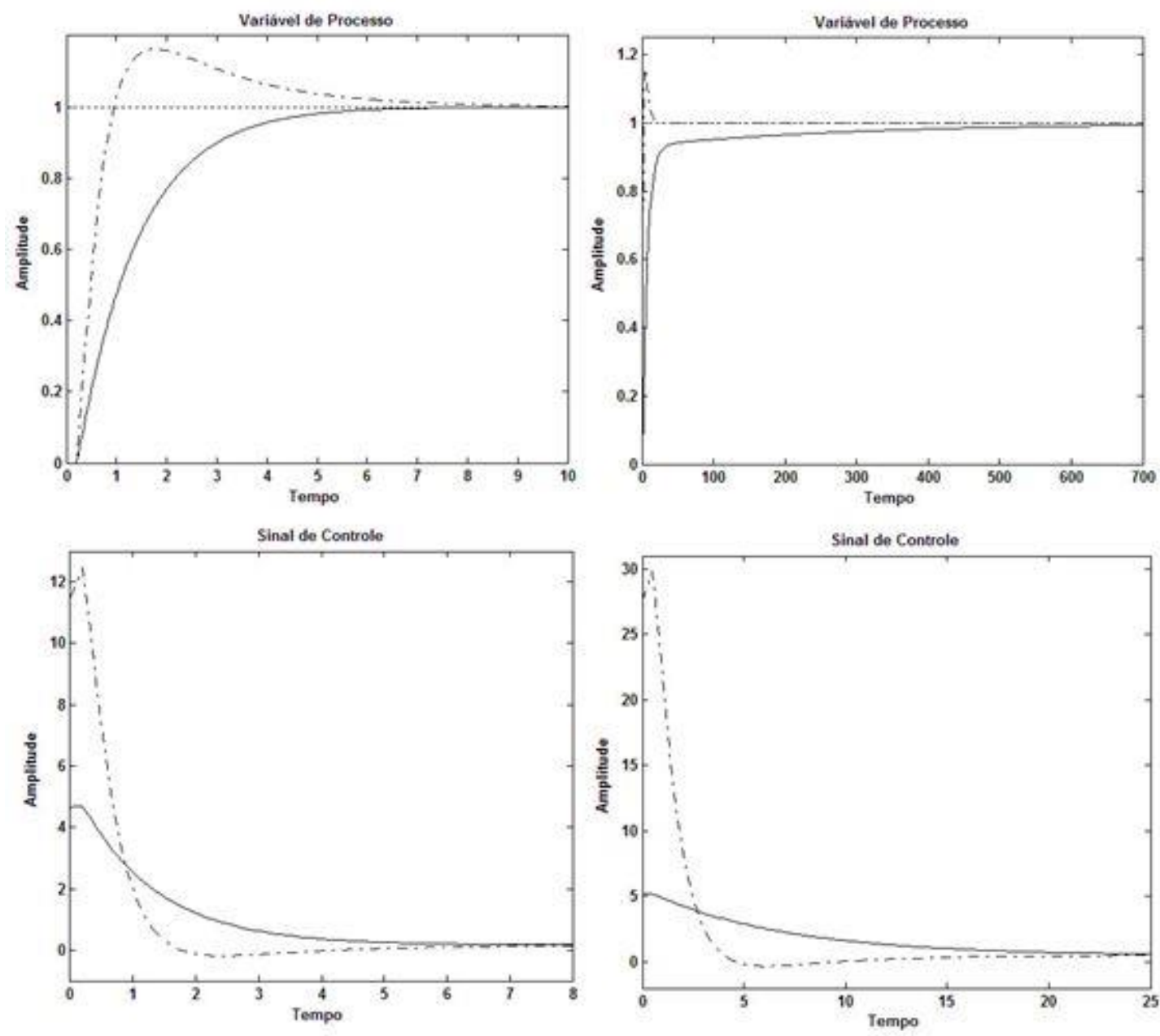

Figura 5. Resposta em malha fechada para um degrau unitário na referência. Modelo $G_{1}(s)$ à esquerda e modelo $\boldsymbol{G}_{2}(\boldsymbol{s})$ à direita. Controlador AMIGO (---) e controlador Proposto (-). Escalas de tempo diferentes para variável de processo e sinal de controle.

\section{DISCUSSÃO}

Como observado nos exemplos 1 e 2 da Seção Resultados, considerar a ação de controle na função de custo $\mathrm{J}$ em (2), foi essencial para minimizar variações rápidas e/ou amplitudes elevadas, se comparada com as sintonias MIGO e AMIGO. Essa importância de considerar o sinal de controle na sintonia de controladores PID se deve ao fato de que atuadores e equipamentos industriais possuem limitações físicas e esse pode não ser realizável na prática. Portanto, a sintonia Proposta produz uma ação de controle mais suave, o que na prática é mais viável, além de reduzir a possibilidade de saturação do atuador, desgaste do equipamento e desestabilizar malhas de controle acopladas. O desempenho fica por conta dos ajustes de $\alpha$ e $\beta$. 


\section{CONCLUSÃO}

Neste trabalho ficou evidenciado a importância de considerar a ação de controle na função de custo, quando utilizado o problema de otimização na sintonia de controladores PID. Exemplos simulados aplicados em modelos lineares são apresentados, no entanto, é possível aplicar esse a modelos não lineares. Além disso, independe se o modelo é estável ou instável. A estabilidade é garantida por restrições no pico das funções sensibilidades.

O uso do AG como ferramenta para solução do problema de otimização apresentou bons resultados com a configuração realizada. No entanto, como o AG é uma técnica de otimização não determinística e sua eficácia é sensível a sua configuração, o AG pode encontrar solução que não seja a global, mas sim local.

\section{Agradecimentos}

Os autores gostariam de agradecer à equipe do Instituto Tecnológico Vale e da Universidade Federal de Ouro Preto pelo apoio no desenvolvimento deste trabalho. Este trabalho foi parcialmente financiado pela CAPES, Vale S.A. e FAPEMIG.

\section{REFERÊNCIAS}

1 Åström KJ, Hägglund T. (2001). The future of PID control. Control Engineering Practice. 2012; 9(11): 1163-1175.

2 Bauer M, Horch A, Xie L, Jelali M, Thornhill N. The current state of control loop performance monitoring - a survey of application in industry. Journal of Process Control. 2016; 38: 1-10.

3 Ziegler JG, Nichols NB. Optimum settings for automatic controllers. Trans. ASME. 1942; 64(11).

4 Hrones KCJ, Reswick J. On the automatic control of the generalized passive systems. Trans. Assoc. Soc. Mech. Eng. 1952.

5 Cohen G, Coon G. Theoretical consideration of retarded control. Trans. ASME. 1953; 75(1): 827-834.

6 Skogestad S. Simple analytic rules for model reduction and PID controller tuning. Journal of process control. 2003; 13(4): 291-309.

7 Campos MCMM, Teixeira HC. Controles típicos de equipamentos e processos industriais. Edgard Blucher; 2006.

8 Pinho AF, Montevechi JAB, Marins FAZ, Miranda RC. Algoritmos genéticos: Fundamentos e aplicações. p.21-32.

9 Herrero JM, Blasco X, Martínez M, Salcedo JV. Optimal PID tuning with genetic algorithms for non linear process models. IFAC Proceedings Volumes. 2002; 35(1): 31-36.

10 Herreros A, Baeyens E, Perán JR. Design of PID-type controllers using multiobjective genetic algorithms. ISA transactions. 2002; 41(4): 457-472.

11 Kristiansson B, Lennartson B. Robust and optimal tuning of PI and PID controllers. IEE Proceedings-Control Theory and Applications. 2002; 149(1):1725.

12 Åström KJ, Panagopoulos H, Hägglund T. Design of pi controllers based on non-convex optimization. Automatica. 1998; 34(5): 585-601.

13 Holland $\mathrm{JH}$. Adaptation in natural and artificial systems. Ann Arbor: The University of Michigan Press. 1975. 
14 Åström KJ, Hägglund T. Advanced PID control. ISA-The Instrumentation, systems and Automation Society. 2006. 